L. GAJeK (Łódź)

P. Miś (Łódź)

J. SŁowińsKa (Amsterdam)

\title{
OPTIMAL STREAMS OF PREMIUMS IN MULTIPERIOD CREDIBILITY MODELS
}

Abstract. Optimal arrangement of a stream of insurance premiums for a multiperiod insurance policy is considered. In order to satisfy solvency requirements we assume that a weak Axiom of Solvency is satisfied. Then two optimization problems are solved: finding a stream of net premiums that approximates optimally 1) future claims, or 2) "anticipating premiums". It is shown that the resulting optimal streams of premiums enable differentiating between policyholders much more quickly than one-period credibility premiums.

1. Introduction. Various aspects of premiums calculation were presented by N. L. Bowers et al. [1], H. U. Gerber [8], H. Bühlmann [2], H. Bühlmann and A. Gisler [3], H. H. Panjer et al. [12], to name but a few authors of monographs only. On the other hand, some authors considered noninsurance approach to optimal funding of a stream of deterministic liabilities (see e.g. [5]). Perhaps the most important difference between insurance and noninsurance approach to financing possible claims is that insurance premiums are calculated for a group of risks in order to cover the total sum of all claims corresponding to those risks. For some insureds the premium calculated in this way will be lower than their future claims and for some it will be higher, but no one is expected to return the resulting profit or to pay an additional post factum premium. If all risks are independent and identically distributed random variables, the system is fair in the sense that it does not privilege any of the insureds. If, however, the risks are not identically distributed, the

2000 Mathematics Subject Classification: 62C10, 62P05, 91B30.

Key words and phrases: multiperiod credibility theory, optimal streams of premiums, progressive solvency requirements, risk profile. 
premium is expected to start differentiating as soon as we get information about the client's claim distribution. Nevertheless even then we still do not expect the client to pay a post factum premium to cover past indemnities; instead we use the information about the past claims to identify better the client's claim distribution. More precisely, according to credibility theory we predict future claims of the client on the basis of his past claims.

In this paper we propose a new method of calculation of insurance premiums when we do not treat each of the insurance periods separately. Such a general approach allows us to propose a more effective way of distributing premiums through all insurance periods. Speaking more technically, every premium is designed so that it takes into account the value of the claims which have happened so far and the values of all claims predicted hereafter. Thus the approach described in the paper may be seen as a generalization of the one-period credibility theory. Premiums calculated in such a way will also ensure a more fair treatment of customers (for details see Section 4).

1.1. The model. Let us consider $m$ insurance periods before the beginning of a new insurance and $T$ periods of the new insurance. The present value of the claim resulting from period $t$ is modeled by a random variable $X_{t}$, where $t=-m, \ldots,-1,1, \ldots, T$. Every client belongs to some class of risk characterized by a risk profile $\theta$ (see [3]). The conditional distribution of each $X_{t}$, given $\theta$, is assumed to be dominated by a $\sigma$-finite measure $\nu$, with a Radon-Nikodym density $w(\cdot \mid \theta)$. The problem is that we usually do not know $\theta$, but basing on historical data we only know that some $\theta$ are more and some are less likely. In the present paper the risk profile $\theta$ is constant in time for each client. It is worth mentioning that $\theta$ may also be time-dependent (see [14] and [3]). Assume that $\Theta$ is a random variable with distribution function $\pi(\theta)$ (also called the structural function of the collective-see [3]). Then the claim $X_{t}$ has the unconditional density function

$$
p(x)=\int w(x \mid \theta) d \pi(\theta) .
$$

Let us define

$$
R_{m}=\sum_{i=-m}^{-1} X_{i}, \quad S_{t}=\sum_{i=1}^{t} X_{i},
$$

for $t=1, \ldots, T$. Assume that

$$
\operatorname{Cov}\left(X_{i}, X_{j} \mid \Theta=\theta\right)= \begin{cases}\sigma^{2}(\theta) & \text { for } i=j \\ 0 & \text { for } i \neq j\end{cases}
$$

and set

$$
\begin{aligned}
& \mathrm{E}\left(X_{i} \mid \Theta=\theta\right)=\mu(\theta), \\
& \mathrm{E}(\mu(\Theta))=\mu, \quad \operatorname{Var}(\mu(\Theta))=a^{2}, \quad \mathrm{E}\left(\sigma^{2}(\Theta)\right)=s^{2},
\end{aligned}
$$


for $i, j=-m, \ldots,-1,1, \ldots, T$. Additionally, we denote by $\beta_{t}\left(x_{-m}, \ldots, x_{-1}\right.$, $\left.x_{1}, \ldots, x_{t-1}\right)$ a prediction of the present value of the client's claim in period $t$ taking into account the values of all his claims in the past. Throughout the paper we shall use the following formula for the best linear predictor, derived in credibility theory (see [3, formula (3.1)]):

$$
\begin{array}{r}
\beta_{t}=\beta_{t}\left(X_{-m}, \ldots, X_{-1}, X_{1}, \ldots, X_{t-1}\right)=z_{t} \frac{S_{t-1}+R_{m}}{t-1+m}+\left(1-z_{t}\right) \mu \\
t=1, \ldots, T
\end{array}
$$

where

$$
z_{t}=\frac{a^{2}(t-1+m)}{s^{2}+a^{2}(t-1+m)}, \quad t=1, \ldots, T .
$$

If $m=0$, we can define $\beta_{t}$ as $\mu$ for $t=1$, and by the right side of (1) with $R_{0}=0$ for $t>1$. The above predictor $\beta_{t}$ is optimal in the sense of minimizing the mean squared error (see e.g. Theorem 3.2 of [3]). However, also other measures are used to find optimal predictors (see e.g. [13]).

Payments of indemnities will be financed by successively collected insurance premiums. The insurance premium in each period will consist of a net premium with present value $P_{t}$ plus premium for the risk, plus the costs and plus return on capital. We define the insurance net premium $P_{t}$ as follows:

$$
\begin{aligned}
P_{t}=\alpha_{t}\left[S_{t-1}+(T-t+1) \beta_{t}\left(X_{-m}, \ldots, X_{-1}, X_{1}, \ldots, X_{t-1}\right)\right] & \\
t & =1, \ldots, T,
\end{aligned}
$$

where $\alpha_{1}, \ldots, \alpha_{T} \geq 0$. The parameter $\alpha_{t}$ defines the fraction of the total of the claims that have happened so far and the ones that are predicted hereafter which the customer finances at period $t$.

1.2. Solvency requirements. The parameter $\alpha_{t}$ is chosen in a way that the following weak Axiom of Solvency be satisfied (see [7] and [4]):

$$
\begin{aligned}
& \mathrm{E} \sum_{i=1}^{t} P_{i} \geq \mathrm{E} \sum_{i=1}^{t} X_{i}, \quad t=1, \ldots, T-1, \\
& \mathrm{E} \sum_{i=1}^{T} P_{i}=\mathrm{E} \sum_{i=1}^{T} X_{i} .
\end{aligned}
$$

By simple algebra, one can show that (3) is equivalent to

$$
\sum_{i=1}^{t} \alpha_{i} \geq \frac{t}{T}, \quad t=1, \ldots, T-1,
$$

and

$$
\sum_{i=1}^{T} \alpha_{i}=1
$$


1.3. The results. Let us observe that

$$
\sum_{t=1}^{T} \mathrm{E}\left(P_{t}-X_{t}\right)^{2}
$$

can be treated as the overall measure of the prediction risk so our aim is to minimize it over all streams $\left(\alpha_{1}, \ldots, \alpha_{T}\right)$ that satisfy (4) and (5). This problem will be solved explicitly in Section 2 (see Theorem 1).

Section 3 will be devoted to finding a stream of insurance premiums well approximating so-called anticipating premiums. This optimization problem is motivated by the fact that if we knew all the claims which were to occur in the future we would be able to calculate the anticipating premiums:

$$
Y_{t}=\alpha_{t} \sum_{i=1}^{T} X_{i}, \quad t=1, \ldots, T,
$$

where the coefficients $\alpha_{t}$ satisfy (4) and (5). Obviously we do not know the claims in advance, therefore we try to find premiums $P_{t}$ which in some sense are close to the anticipating premiums $Y_{t}$. Theorem 2 says that the weights

$$
\alpha_{t}=1 / T, \quad t=1, \ldots, T,
$$

provide such an approximation. Moreover, the above constant weights are the limits, as $s / \mu \rightarrow 0$, of the weights solving the first optimization problem. The main idea of this paper is based on the results of [5], [11] and [15].

It is also possible to look at the credibility theory from other points of view. For instance, Landsman [9] considers the second order optimal estimation while Bühlmann and Gisler [3] investigate time-dependent risk profiles of the insureds. For other approaches and extensions we refer the reader to the monograph of Bühlmann and Gisler [3].

2. Insurance premiums approximating claims. Let us suppose that the net insurance premiums should correspond as well as possible to the future payment in every period. This leads to the following optimization problem:

$$
\underset{\alpha_{1}, \ldots, \alpha_{T}}{\operatorname{minimize}} \sum_{t=1}^{T} \mathrm{E}\left(P_{t}-X_{t}\right)^{2}
$$

subject to

$$
\begin{cases}\sum_{i=1}^{t} \alpha_{i} \geq t / T, & t=1, \ldots, T-1, \\ \alpha_{t} \geq 0, & t=1, \ldots, T, \\ \sum_{t=1}^{T} \alpha_{t}=1, & \end{cases}
$$

where constraints (8) correspond to solvency restrictions (3). 
Optimal coefficients $\alpha_{t}$ that solve (7) subject to (8) are provided in Theorem 1 below.

THEOREM 1. If

$$
\alpha_{t}=\left(\frac{1-\sum_{i=1}^{T} l_{i} / k_{i}}{\sum_{i=1}^{T} 1 / k_{i}}+l_{t}\right) \frac{1}{k_{t}}, \quad t=1, \ldots, T,
$$

where $k_{i}$ and $l_{i}$ are given by (11) and (12) below, then $\left(\alpha_{1}, \ldots, \alpha_{T}\right)$ is a solution to (7) subject to (8).

Proof. Let us notice that

$$
\begin{aligned}
\sum_{t=1}^{T} \mathrm{E}\left(P_{t}-\right. & \left.X_{t}\right)^{2} \\
= & \sum_{t=1}^{T} \frac{\alpha_{t}^{2}}{s^{2}+a^{2}(t-1+m)}\left[(t-1) s^{4}+(2 T+m)(t-1) a^{2} s^{2}\right. \\
& \left.+T^{2} a^{4}(t-1+m)+T^{2} \mu^{2}\left((t-1+m) a^{2}+s^{2}\right)\right] \\
& -2 \sum_{t=1}^{T} \frac{\alpha_{t}}{s^{2}+a^{2}(t-1+m)}\left[(t-1) s^{2} a^{2}+T(t-1+m) a^{4}\right. \\
& \left.+T \mu^{2}\left(s^{2}+a^{2}(t-1+m)\right)\right]+T\left(a^{2}+s^{2}+\mu^{2}\right) .
\end{aligned}
$$

It is easy to show that the foregoing function is convex. Let us define

$$
\begin{aligned}
k_{t}= & \frac{1}{s^{2}+a^{2}(t-1+m)}\left[(t-1) s^{4}+(2 T+m)(t-1) a^{2} s^{2}\right. \\
& \left.+T^{2} a^{4}(t-1+m)+T^{2} \mu^{2}\left((t-1+m) a^{2}+s^{2}\right)\right], \\
l_{t}= & \frac{(t-1) s^{2} a^{2}+T(t-1+m) a^{4}+T \mu^{2}\left(s^{2}+a^{2}(t-1+m)\right)}{s^{2}+a^{2}(t-1+m)},
\end{aligned}
$$

and

$$
A=T\left(a^{2}+s^{2}+\mu^{2}\right) .
$$

Then the Lagrangian for our problem can be written as follows:

$$
\begin{aligned}
L(\boldsymbol{\alpha}, \boldsymbol{\lambda}, p)= & \sum_{t=1}^{T}\left(\alpha_{t}^{2} k_{t}-2 \alpha_{t} l_{t}\right)+A+\sum_{t=1}^{T-1} \lambda_{t}\left(\frac{t}{T}-\sum_{i=1}^{t} \alpha_{i}\right) \\
& +p\left(1-\sum_{i=1}^{T} \alpha_{i}\right) .
\end{aligned}
$$

By the Kuhn-Tucker theorem (see [12, Corollary 8.11.2]), the optimization problem (7)-(8) takes the form 


$$
\begin{cases}2 \alpha_{t} k_{t}-2 l_{t}-\sum_{i=t}^{T-1} \lambda_{i}-p=0, & t=1, \ldots, T, \\ \lambda_{t}\left(\frac{t}{T}-\sum_{i=1}^{t} \alpha_{i}\right)=0, & t=1, \ldots, T-1, \\ 1-\sum_{i=1}^{T} \alpha_{i}=0, & \\ \frac{t}{T}-\sum_{i=1}^{t} \alpha_{i} \leq 0, & t=1, \ldots, T-1, \\ \alpha_{t} \geq 0, & t=1, \ldots, T, \\ p \in \mathbb{R}, & t=1, T-1 . \\ \lambda_{t} \geq 0, & \end{cases}
$$

Assume that $\lambda_{1}=\cdots=\lambda_{T-1}=0$ and $\alpha_{1}, \ldots, \alpha_{T}, p>0$. Then from the first equation of (13) we obtain

$$
\alpha_{t}=\frac{2 l_{t}+p}{2 k_{t}}, \quad t=1, \ldots, T,
$$

and additionally, for $t=1$,

$$
p=2 \alpha_{1} k_{1}-2 l_{1} \text {. }
$$

Thus

$$
\alpha_{t}=\frac{l_{t}+\alpha_{1} k_{1}-l_{1}}{k_{t}}, \quad t=1, \ldots, T .
$$

Then, using the third equation of (13), we get

$$
\sum_{i=1}^{T} \frac{l_{i}-l_{1}+\alpha_{1} k_{1}}{k_{i}}=1
$$

hence

$$
\alpha_{1}=\frac{1-\sum_{i=1}^{T}\left(l_{i}-l_{1}\right) / k_{i}}{\sum_{i=1}^{T} k_{1} / k_{i}} .
$$

From (14) the optimal choice of $\alpha_{t}$ is

$$
\alpha_{t}=\left(\frac{1-\sum_{i=1}^{T} l_{i} / k_{i}}{\sum_{i=1}^{T} 1 / k_{i}}+l_{t}\right) \frac{1}{k_{t}}>0, \quad t=1, \ldots, T,
$$

and

$$
p=2 \frac{1-\sum_{i=1}^{T} l_{i} / k_{i}}{\sum_{i=1}^{T} 1 / k_{i}}>0 .
$$

It is easy to check that

$$
l_{t} / k_{t}>l_{t+1} / k_{t+1}, \quad k_{t}<k_{t+1} \quad t=1, \ldots, T-1 .
$$


Therefore

$$
\alpha_{t}>\alpha_{t+1}, \quad t=1, \ldots, T-1 .
$$

From (15) and the third equation of (13) we have

$$
\sum_{i=1}^{t} \alpha_{i} \geq t / T \quad t=1, \ldots, T-1 .
$$

Thus we have obtained the coefficients $\alpha_{t}$ solving (7) subject to (8).

Theorem 1 enables us to construct the stream of insurance premiums which well approximates the stream of claims. Formula (9) simplifies considerably for some special limit case. Notice that if $s \ll \mu$ (so that $s^{2} / \mu^{2} \approx 0$ ), from (11) and (12) we get

$$
\frac{l_{t}}{k_{t}} \frac{\mu^{-2}}{\mu^{-2}} \approx \frac{T(t-1+m) a^{4} / \mu^{2}+T\left(s^{2}+a^{2}(t-1+m)\right)}{T^{2}(t-1+m) a^{4} / \mu^{2}+T^{2}\left(s^{2}+a^{2}(t-1+m)\right)}=\frac{1}{T} .
$$

Therefore, by (9),

$$
\alpha_{t}=\left(\frac{1-\sum_{i=1}^{T} \frac{l_{i}}{k_{i}} \frac{\mu^{-2}}{\mu^{-2}}}{\sum_{i=1}^{T} \frac{1}{k_{i}}}+l_{t}\right) \frac{1}{k_{t}} \approx\left(\frac{1-\sum_{i=1}^{T} \frac{1}{T}}{\sum_{i=1}^{T} \frac{1}{k_{i}}}+l_{t}\right) \frac{1}{k_{t}}=\frac{1}{T} .
$$

We will show in Section 3 that the above limit case premiums have further optimality properties.

3. Anticipating insurance premiums. If we could know the future, an insured should pay the total net premium $\sum_{i=1}^{T} X_{i}$. This total premium could be distributed over all insurance periods in such a way that the weak Axiom of Solvency (3) be satisfied. So let us define

$$
Y_{t}=\alpha_{t} \sum_{i=1}^{T} X_{i}, \quad t=1, \ldots, T,
$$

where the coefficients $\alpha_{t}$ satisfy (4) and (5). Throughout the paper the random variables $Y_{t}$ are called the anticipating premiums. They could be calculated if we were able to use in the premium formula both the claims that happened in the past and those which will occur hereafter. Suppose that we want to minimize the overall difference between the insurance premiums $P_{t}$, given by (2), and the anticipating premiums $Y_{t}$. To be more precise, we are looking for a solution to the following problem:

$$
\underset{\alpha_{1}, \ldots, \alpha_{T}}{\operatorname{minimize}} \sum_{t=1}^{T} \mathrm{E}\left(P_{t}-Y_{t}\right)^{2},
$$

subject to 


$$
\begin{cases}\sum_{i=1}^{t} \alpha_{i} \geq t / T, & t=1, \ldots, T-1, \\ \alpha_{t} \geq 0, & t=1, \ldots, T, \\ \sum_{t=1}^{T} \alpha_{t}=1 . & \end{cases}
$$

THEOREM 2. The weights

$$
\alpha_{t}=1 / T, \quad t=1, \ldots, T
$$

solve the minimization problem (16) subject to (17).

Proof. Let us notice that

$$
\sum_{t=1}^{T} \mathrm{E}\left(P_{t}-Y_{t}\right)^{2}=\sum_{t=1}^{T} \alpha_{t}^{2} \frac{s^{2}+(T+m) a^{2}}{s^{2}+a^{2}(t-1+m)}(T-t+1) s^{2} .
$$

It is easily seen that the above function is convex. Let us define

$$
k_{t}=\frac{s^{2}+(T+m) a^{2}}{s^{2}+a^{2}(t-1+m)}(T-t+1) s^{2} .
$$

The Lagrangian for our problem is given by

$$
\begin{aligned}
L(\boldsymbol{\alpha}, \boldsymbol{\lambda}, p)= & \sum_{t=1}^{T} \alpha_{t}^{2} k_{t}+\sum_{t=1}^{T-1} \lambda_{t}\left(\frac{t}{T}-\sum_{i=1}^{t} \alpha_{i}\right) \\
& +p\left(1-\sum_{i=1}^{T} \alpha_{i}\right) .
\end{aligned}
$$

By the use of the Kuhn-Tucker theorem (see [12, Corollary 8.11.2]), the minimization problem (16)-(17) takes the form

$$
\begin{cases}2 \alpha_{t} k_{t}-\sum_{i=t}^{T-1} \lambda_{i}-p=0, & t=1, \ldots, T, \\ \lambda_{t}\left(\frac{t}{T}-\sum_{i=1}^{t} \alpha_{i}\right)=0, & t=1, \ldots, T-1, \\ 1-\sum_{i=1}^{T} \alpha_{i}=0, & \\ \frac{t}{T}-\sum_{i=1}^{t} \alpha_{i} \leq 0, & t=1, \ldots, T-1, \\ \alpha_{t} \geq 0, & t=1, \ldots, T, \\ p \in \mathbb{R}, & \\ \lambda_{t} \geq 0, & \end{cases}
$$


Assume that $\alpha_{1}, \ldots, \alpha_{T}, p>0$. From the first equation of (18) we get

$$
\alpha_{t}=\frac{\sum_{i=t}^{T-1} \lambda_{i}+p}{2 k_{t}}, \quad t=1, \ldots, T \text {. }
$$

Note that for $t=T$,

$$
p=2 k_{t} \alpha_{T}
$$

Also assume that $\lambda_{t}>0$ for $t=1, \ldots, T-1$. Then from the second equation of (18) we obtain

$$
\alpha_{t}=1 / T>0, \quad t=1, \ldots, T
$$

and

$$
p=2 k_{T} / T>0
$$

Thus

$$
\frac{1}{T}=\alpha_{t}=\frac{\lambda_{t}+\sum_{i=t+1}^{T-1} \lambda_{i}+p}{2 k_{t}}, \quad t=1, \ldots, T
$$

hence

$$
\begin{aligned}
\lambda_{t} & =\frac{2 k_{t}}{T}-\sum_{i=t+1}^{T-1} \lambda_{i}-p=\left(\frac{2 k_{t}}{T}-p-\sum_{i=t+2}^{T-1} \lambda_{i}\right)-\lambda_{t+1} \\
& =\left(\frac{2 k_{t}}{T}-p-\sum_{i=t+2}^{T-1} \lambda_{i}\right)-\left(\frac{2 k_{t+1}}{T}-\sum_{i=t+2}^{T-1} \lambda_{i}-p\right)=\frac{2\left(k_{t}-k_{t+1}\right)}{T}
\end{aligned}
$$

for all $t=1, \ldots, T$. It is a simple matter to show that

$$
k_{t}>k_{t+1}, \quad t=1, \ldots, T-1 \text {. }
$$

Therefore

$$
\lambda_{t}=\frac{2\left(k_{t}-k_{t+1}\right)}{T}>0, \quad t=1, \ldots, T-1
$$

Hence

$$
\alpha_{t}=1 / T, \quad t=1, \ldots, T,
$$

minimize (16). It can be easily checked that the $\alpha_{t}$ given by (19) satisfy restrictions (17) so they provide the solution to our problem. Thus we have constructed the insurance premiums

$$
P_{t}=\frac{1}{T}\left[S_{t-1}+(T-t+1)\left(z_{t} \frac{S_{t-1}+R_{m}}{t-1+m}+\left(1-z_{t}\right) \mu\right)\right], \quad t=1, \ldots, T
$$

where $z_{t}=a^{2}(t-1+m) /\left[s^{2}+a^{2}(t-1+m)\right]$, which are closest, in the sense of (16) and (17), to the anticipating premiums.

4. Comparisons with one-period credibility premiums. Let us compare our methodology with the one corresponding to the one-period credibility theory. First observe that for each $t=1, \ldots, T$, the predictor 
$\beta_{t}$ equals the credibility premium. Let us suppose that $\alpha_{t}=1 / T$ for all $t=1, \ldots, T$. The function

$$
U_{t}(\theta)=\mathrm{E}\left[P_{t}-\beta_{t} \mid \Theta=\theta\right], \quad t=1, \ldots, T,
$$

measures expected differences between the premium's values when applying our approach and those given by the one-period credibility theory, for an arbitrary fixed risk profile $\theta$. It is easy to check that

$$
U_{t}(\theta)=\frac{s^{2}}{T\left[s^{2}+a^{2}(t-1+m)\right]}\left[\sum_{i=1}^{t-1} \mathrm{E}\left(X_{i} \mid \Theta=\theta\right)-(t-1) \mu\right] .
$$

Notice that $U_{1}(\theta)=0$. This means that the insurance premiums are equal to each other for both approaches in the first period. Now, let us consider the case $t>1$.

Corollary 3.

$$
\begin{array}{ll}
U_{t}(\theta)>0 & \text { if } \mathrm{E}(X \mid \Theta=\theta)>\mu(\text { bad risk case }), \\
U_{t}(\theta)=0 & \text { if } \mathrm{E}(X \mid \Theta=\theta)=\mu(\text { average risk case }), \\
U_{t}(\theta)<0 & \text { if } \mathrm{E}(X \mid \Theta=\theta)<\mu(\text { good risk case })
\end{array}
$$

for every $t>1$.

This means that bad risks pay higher premiums in our model, average risks have the same premiums in both models, and good risks pay lower premiums. Additionally we have

Corollary 4.

$$
\text { If } \mathrm{E}(X \mid \Theta=\theta)>\mu \text { (bad risk case), then } U_{t+1}>U_{t}>0 \text {, }
$$

and

$$
\text { if } \mathrm{E}(X \mid \Theta=\theta)<\mu(\text { good risk case }) \text {, then } U_{t+1}<U_{t}<0
$$

for all $t>1$.

Let us notice that bad risks will pay higher and higher premiums, compared with the one-period credibility theory, and good risks will pay lower and lower ones. More detailed comparisons are provided in the following example.

EXAmPle 5. Assume that the claims have Gamma distribution with parameters $p, \theta$ :

$$
w(x \mid \theta)=\frac{\theta^{p}}{\Gamma(p)} x^{p-1} e^{-\theta x},
$$

and $\Theta$ is a random variable with a density function 


$$
\pi(\theta)=\frac{d^{c}}{\Gamma(c)} \theta^{c-1} e^{-d \theta} .
$$

We take $T=10$ and $m=4$. Additionally let $p=1, c=21$ and $d=4000$. From the above it follows that

$$
\begin{aligned}
\mathrm{E}(\mu(\Theta)) & =\mu=\$ 200, \\
\operatorname{Var}(\mu(\Theta)) & =a^{2}=2105.26, \\
\mathrm{E}\left(\sigma^{2}(\Theta)\right) & =s^{2}=42105.26 .
\end{aligned}
$$

Let us consider three policyholders: insured A with $\mathrm{E}(X \mid \Theta=\theta)=\$ 108$ (good risk case), insured $\mathrm{B}$ with $\mathrm{E}(X \mid \Theta=\theta)=\$ 200$ (average risk case), and insured $\mathrm{C}$ with $\mathrm{E}(X \mid \Theta=\theta)=\$ 292$ (bad risk case).

Table 1. Expected differences between multiand one-period credibility net premiums

\begin{tabular}{lccc}
\hline Period & Good risk & Average risk & Bad risk \\
$t$ & $U_{t}(\theta)$ & $U_{t}(\theta)$ & $U_{t}(\theta)$ \\
& $(\$)$ & $(\$)$ & $(\$)$ \\
\hline 1 & -0.00 & 0.00 & 0.00 \\
2 & -7.36 & 0.00 & 7.36 \\
3 & -14.15 & 0.00 & 14.15 \\
4 & -20.44 & 0.00 & 20.44 \\
5 & -26.29 & 0.00 & 26.29 \\
6 & -31.72 & 0.00 & 31.72 \\
7 & -36.80 & 0.00 & 36.80 \\
8 & -41.55 & 0.00 & 41.55 \\
9 & -46.00 & 0.00 & 46.00 \\
10 & -50.18 & 0.00 & 50.18 \\
\hline Total & -274.50 & 0.00 & 274.50 \\
\hline
\end{tabular}

Thus, due to our methodology after 10 periods insured A (good risk) saves on average $\$ 274.50$ while insured C (bad risk) pays on average $\$ 274.50$ more.

In Figure 1 it is shown how the average premiums change in time for good and bad risks as compared with the one-period credibility theory premiums. In our model in the last period a bad risk pays over $\$ 50$ more and a good risk pays over $\$ 50$ less than in the one-period credibility theory. In our model the average total sum of premiums of insured A (good risk) is $\$ 1457.79$. Insured A can buy for this sum only 8 premiums according to the one-period credibility theory.

In Figure 2 it is shown in successive periods how $U_{t}(\theta)$ changes in relation to $\mathrm{E}\left[\beta_{t} \mid \Theta=\theta\right]$. In period 10 a bad risk pays the premium $21 \%$ higher 

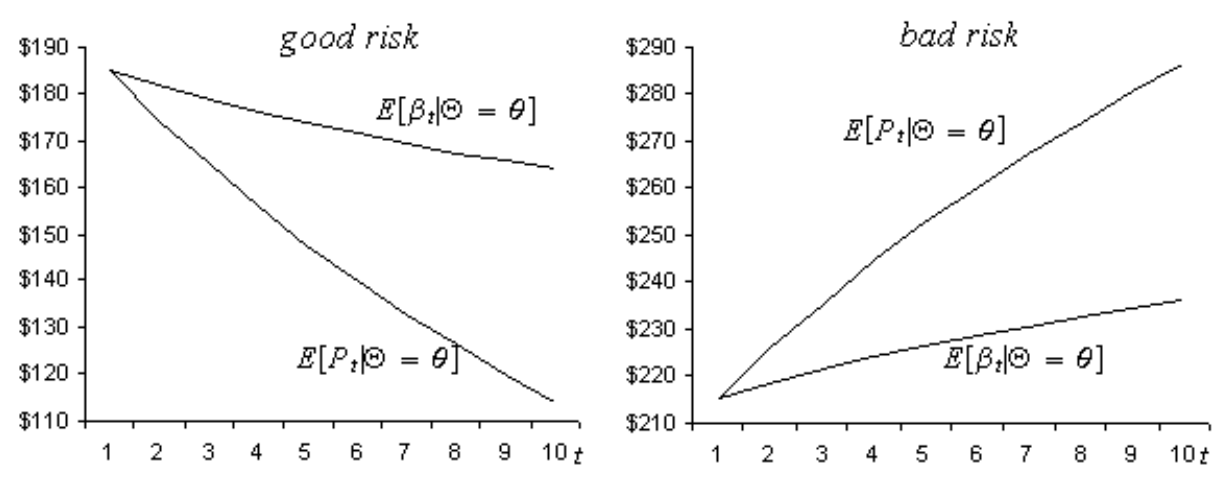

Fig. 1. Expected net premiums of good and bad risks in a multiperiod credibility model (compared with one-period credibility premiums).

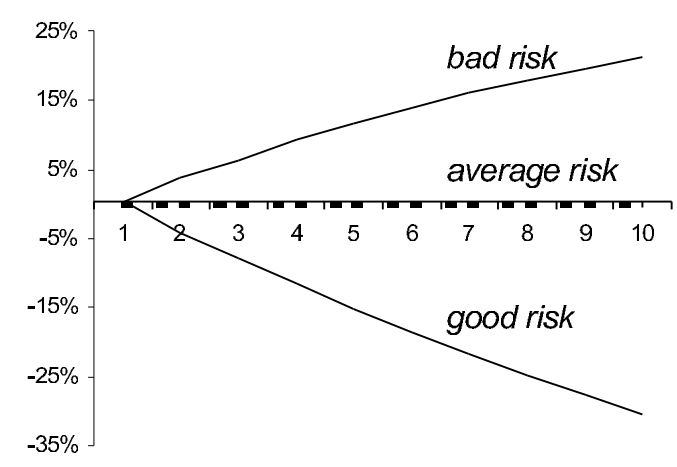

Fig. 2. Expected difference $U_{t}(\theta)$ as a percentage of net premiums in a one-period credibility model.

and a good risk pays the premium $31 \%$ lower than given by the one-period credibility theory.

The above example shows the advantage of the multiperiod credibility approach: it is profitable for good risks and unprofitable for bad ones. Thus good risks would be more likely to choose our company whereas bad risks would rather go to other ones. Therefore we could lower the premiums successively while the competing companies will have to increase their premiums in order to cover an increasing number of bad risks.

\section{References}

[1] N. L. Bowers, H. U. Gerber, I. C. Hickman, D. A. Jones and C. J. Nesbitt, Actuarial Mathematics, 2nd ed., Society of Actuaries, Schaumburg, IL, 1996.

[2] H. Bühlmann, Mathematical Methods of Risk Theory, Springer, Berlin, 1970. 
[3] H. Bühlmann and A. Gisler, A Course in Credibility Theory and Its Applications, Springer, Berlin, 2005.

[4] L. Gajek, Axiom of Solvency and portfolio immunization under random interest rates, Insurance Math. Econom. 36 (2005), 317-328.

[5] L. Gajek and K. Ostaszewski, Optimal funding of a liability, J. Insurance Issues 24 (2001), 17-29.

[6] -, 一, Pension Plans. Asset-Liability Management, WNT, Warszawa, 2002 (in Polish).

[7] —, - Financial Risk Management for Pension Plans, Elsevier, Amsterdam, 2004.

[8] H. U. Gerber, An Introduction to Mathematical Risk Theory, S. S. Heubner Found. Monogr. Ser. 8, Richard D. Irwin Inc., Homewood, IL, 1979.

[9] Z. Landsman, Credibility theory: a new view from the theory of second order optimal statistics, Insurance Math. Econom. 30 (2002), 251-262.

[10] M. Mashayekhi, On asymptotic optimality in empirical Bayes credibility, ibid. 31 (2002), 285-295.

[11] P. Miś, Optimal designing of a sequence of premiums for long-term insurance policies, Diploma Thesis, Inst. Math., Łódź Technical Univ., Łódź, 2002 (in Polish).

[12] H. H. Panjer (ed), Financial Economics: With Applications to Investments, Insurance and Pensions, The Actuarial Foundation, Schaumburg, IL, 1998.

[13] S. D. Promislow and V. R. Young, Equity and exact credibility, Astin Bull. 30 (2000), $3-11$.

[14] O. Purcaru and M. Denuit, Dependence in dynamic claim frequency credibility models, ibid. 33 (2003), 23-40.

[15] J. Słowińska, Optimization of premiums in a multiperiod insurance, Diploma Thesis, Faculty of Mathematics, Informatics and Mechanics, Warsaw Univ., Warszawa, 2004 (in Polish).

Lesław Gajek

Institute of Mathematics

Technical University of Łódź

Wólczańska 215

90-924 Łódź, Poland

E-mail: gal@p.lodz.pl

and

Institute of Applied Mathematics

and Mechanics

Warsaw University

Banacha 2

02-097 Warszawa, Poland
Paweł Miś

Institute of Mathematics Technical University of Łódź Wólczańska 215 90-924 Łódź, Poland E-mail: pmis@im0.p.lodz.pl

Joanna Słowińska Computer System Section Department of Computer Science

Faculty of Science

Vrije Universiteit

De Boelelaan 1081

1081 HV Amsterdam, The Netherlands

E-mail: asia@few.vu.nl 
